\title{
The effects of prior knowledge and text structure on comprehension processes during reading of scientific texts
}

\author{
Panayiota Kendeou \\ McGill University, Montreal, Quebec, Canada \\ AND \\ Paul VAN Den Broek \\ University of Minnesota, Minneapolis, Minnesota
}

\begin{abstract}
The aim of the present study was to investigate the effects of prior knowledge and text structure on cognitive processes during comprehension of scientific texts. To investigate the processes online, we used a thinkaloud methodology in Experiment 1 and a reading time methodology in Experiment 2. In both experiments, we obtained offline comprehension measures and measures of individual differences in working memory and need for cognition. Across the two experiments, the results indicated that readers adjust their processing as a function of the interaction between prior knowledge and text structure. In particular, adjustments in the actual processes that take place during reading were observed for readers who had erroneous prior knowledge, but only when they read a text that was structured to explicitly refute this prior knowledge. Furthermore, the results showed that readers' memory for the text was affected by differences in their prior knowledge, independently of text structure. These findings contribute to our understanding of the relation between different factors that are associated with comprehension of scientific texts and have implications for theories of comprehension and conceptual change.
\end{abstract}

Many factors affect success or failure during comprehension of expository texts. Reader characteristics, text properties, and the instructional context in which reading takes place are just a few of them (Goldman \& Bisanz, 2002; Jenkins, 1979; van den Broek, Fletcher, \& Risden, 1993; van den Broek \& Kremer, 1999). Although these factors have often been studied in isolation, it is their interactions and interdependencies that provide important information about naturalistic text comprehension (Rapp \& van den Broek, 2005). In the present study, we focus on two factors: reader characteristics and text properties. The goal is to investigate the possible interactions between readers' prior knowledge and the structure of the text in the comprehension processes that occur during reading of scientific texts.

There is ample evidence that the quantity or amount of readers' prior knowledge influences comprehension of scientific texts (see, e.g., Chi, 1978; Chi, Feltovich, \& Glaser, 1981). Indeed, there is a well-documented advantage in comprehension of texts for readers with high knowledge on the topics described by the texts over readers with low knowledge (e.g., Bartlett, 1932; Chiesi, Spilich, \& Voss, 1979; Dochy, Segers, \& Buehl, 1999; Means \& Voss, 1985; Recht \& Leslie, 1988). In the present study, we focus on readers' quality of knowledge. Quality refers to the accuracy of one's knowledge and has been investigated mostly with respect to students' inaccurate ideas in science (Kendeou, Rapp, \& van den Broek, 2004; Kendeou $\&$ van den Broek, 2005). Inaccurate ideas, or misconceptions, have been found to interfere with the acquisition of new, related knowledge from texts (Alvermann, Smith, \& Readence, 1985; Diakidoy \& Kendeou, 2001; Lipson, 1982; Maria \& MacGinitie, 1987; Peeck, van den Bosch, \& Kreupeling, 1982). The evidence for interference with learning comes from offline studies - that is, studies in which the end product of reading is assessed by tasks such as recall or question answering, but the presumption is that the interference occurs during the reading process itself. How the processes during reading are influenced by misconceptions has not been studied directly, however, and therefore the details of how misconceptions affect reading are not known.

A second factor that influences comprehension of information in scientific texts is the structure of the text. There are different ways for an author to organize the ideas in texts in general or in scientific texts in particular, resulting in different structures (Graesser, Leon, \& Otero, 2002). Meyer and colleagues (Meyer, 1975, 1999; Meyer

P. Kendeou, kend0040@umn.edu 
\& Freedle, 1984) demonstrated that readers' comprehension is influenced by the text structure used to convey the information. In the present study, we focus on a text structure that is often used in organizing the material in scientific texts, refutation texts. Refutation texts are used primarily to persuade students to change prior beliefs by explicitly identifying misconceptions and explaining the correct ideas (Alvermann \& Hague, 1989; Chambliss, 2002; Guzzetti, Snyder, Glass, \& Gamas, 1993). Such texts have been tested in science education literature and have been found to be effective in helping students change their misconceptions and engage in conceptual change (Alvermann \& Hynd, 1989; Diakidoy, Kendeou, \& Ioannides, 2003; Maria \& MacGinitie, 1987).

It is likely that in most instances, prior knowledge and text structure interact in their effects on readers' comprehension processes. Indeed, there is evidence that the interaction between readers' prior knowledge and text characteristics influences comprehension once reading is completed. For example, using offline tasks such as recall and inferential and problem-solving questions, McNamara and colleagues have demonstrated that high-knowledge readers' comprehension was better for low- than for highcohesion texts, whereas low-knowledge readers' comprehension was better for high- than for low-cohesion texts (McNamara, 2001; McNamara \& Kintsch, 1996; McNamara, Kintsch, Songer, \& Kintsch, 1996). The findings of these studies suggest that the interaction between prior knowledge and text characteristics affects comprehension, at least when it is measured after reading has been completed. The implicit assumption is, however, that the interaction between prior knowledge and text characteristics affects the offline product of reading by affecting the online processes that take place during reading (e.g., Goldman \& Varma, 1995; Kintsch, 1988; Trabasso \& Suh, 1993; van den Broek, Rapp, \& Kendeou, 2005; van den Broek, Young, Tzeng, \& Linderholm, 1999; Zwaan \& Singer, 2003). In the present study, we directly investigate this assumption.

The aim of our study is to investigate the effects of readers' prior knowledge, as well as text structure, and the possible interaction of the two, online - that is, during comprehension of text. We focus particularly on cognitive processing of refutation and nonrefutation scientific texts by readers with and without misconceptions related to the topics of the text. We also take into account other individual differences, such as working memory capacity (Baddeley \& Hitch, 1974; Budd, Whitney, \& Turley, 1995) and need for cognition (Cacioppo \& Petty, 1982), because these differences are commonly considered important in many reading situations. For instance, reading a text while having misconceptions may result in interference, because readers need to reconcile the contradiction between what they already know and what is presented in the text. In tasks that involve interference and the need to reconcile different sources of information, working memory is likely to play a role (Engle, 2002; Engle \& Conway, 1998). Also, enjoyment of thinking and engagement - the two main characteristics of need for cognition - are strongly related to reading comprehension (Guthrie, McGough,
Bennett, \& Rice, 1996). Thus, it is likely that readers' need for cognition plays a role when they need to resolve inconsistencies between their misconceptions and information presented in the text during comprehension (Kardash \& Scholes, 1996).

To address these issues, we conducted two experiments. In Experiment 1, we used a think-aloud methodology to investigate cognitive processes online. This methodology allows the consideration of a variety of readers' responses (Ericsson \& Simon, 1993; Pressley \& Afflerbach, 1995; Trabasso \& Suh, 1993) and has received extensive validation as a tool to reveal comprehension processes in reading (Afflerbach, 2002; Coté \& Goldman, 1999; Magliano \& Graesser, 1991; Magliano \& Millis, 2003; Magliano, Trabasso, \& Graesser, 1999; Zwaan \& Brown, 1996). In Experiment 2, we used a reading time methodology to investigate the cognitive processes online, because it is unobtrusive and reflects both strategic and automatic processes. Thus, the methods used in the two experiments complemented each other and provided the basis for acquiring convergent evidence for readers' cognitive processing. In both experiments, we obtained offline comprehension measures and measures of readers' individual differences in prior knowledge, working memory, and need for cognition.

\section{EXPERIMENT 1 Think-Aloud Methodology}

The objective of Experiment 1 was to investigate the effects of prior knowledge and text structure online, using a think-aloud methodology. In this experiment, participants with and without misconceptions in physics were asked to read a refutation and a nonrefutation scientific text while performing a think-aloud task. Participants were also asked to recall the texts they read and to complete measures of working memory capacity and need for cognition.

Given the evidence from offline tasks that refutation texts are particularly effective in bringing about learning in readers with misconceptions (e.g., Guzzetti et al., 1993), we expected that the interaction between readers' prior knowledge and text structure would affect readers' online comprehension processes. First, we expected that readers with misconceptions would engage in conceptual change processes during reading of the refutation text structure more so than during reading of nonrefutation text, and more so than their counterparts without misconceptions for either text structure. Second, we expected that readers' prior knowledge would affect their online comprehension processes, particularly the content of those processes that involve prior knowledge. Thus, we expected that readers with misconceptions would generate more incorrect knowledge-based inferences than would readers without misconceptions, whereas readers without misconceptions would generate more correct knowledge-based inferences than would readers with misconceptions. A third prediction pertains to the connection between online reading processes and offline reading outcome. Given that the online processes form the basis for the construction of a memory 
representation (Goldman \&Varma, 1995; Kintsch, 1988; Langston \& Trabasso, 1999; Myers \& O’Brien, 1998; van den Broek et al., 1999; Zwaan, 1999), we predicted that the patterns of online processes that we obtained would be reflected in the patterns of results for the offline memory task. That is, we expected that higher order cognitive processing (e.g., inference making) would result in better comprehension (e.g., higher recall).

\section{Method}

\section{Participants}

A total of 86 University of Minnesota undergraduates enrolled in introductory psychology or physics courses participated in this study. Students received extra course credit or a gift certificate for their participation. The data of 6 students were eliminated from the study because of equipment failure ( 2 students), or because they were nonnative English speakers (4 students). Of the remaining participants, 41 were female and 39 were male, with an age range of $18-48$ years.

\section{Materials}

Identification of misconceptions. To diagnose readers' misconceptions in Newtonian mechanics, we used the Force Concept Inventory (Hestenes, Wells, \& Swackhamer, 1992). The inventory includes a total of 30 multiple-choice questions in six categories: kinematics, Newton's first law, Newton's second law, Newton's third law, superposition, and kinds of force. Each question has one correct answer, with the remainder of the answers corresponding to commonsense misconceptions of Newtonian concepts. The participants were divided into two groups, misconception and nonmisconception, following the procedures recommended by Hestenes and colleagues (Hestenes \& Halloun, 1995; Hestenes et al., 1992). The misconception group was made up of participants who scored $60 \%$ or less on the inventory ( 30 females, 10 males). Participants in this range have been described as holding incoherent ideas and misconceptions related to Newtonian mechanics (Hestenes et al., 1992). The nonmisconception group was made up of participants who scored above $60 \%$ on the inventory ( 11 females, 29 males). In prior research (Hestenes et al., 1992), participants in this range have been determined to hold coherent ideas, including the concepts of velocity, acceleration, and force.

Working memory capacity. To assess readers' working memory capacity, we used a reading span test (Singer, Andrusiak, Reisdorf, \& Black, 1992). In this test, participants are asked to read sets of unrelated sentences one at a time on the computer. The sets begin in groupings of two sentences and increase to five sentences per set. Each sentence in a set appears for $7 \mathrm{sec}$ on the screen. After reading each set, participants are prompted by a chime to write down the last word of each sentence they read. Finally, they complete a cloze task by writing two missing words from one of the sentences. The cloze task is intended to prevent readers from adopting a strategy of focusing on and memorizing only the final words. The total number of final words recalled correctly constitutes the reader's working memory capacity score. The maximum score a participant could achieve was 46

Need for Cognition Scale. To assess readers' enjoyment of thinking and engagement, we used the Need for Cognition Scale (Cacioppo \& Petty, 1982). The instrument consists of 18 statements, for which students rate the degree to which each statement characterizes them (using a 1-5 Likert scale, ranging from extremely uncharacteristic to extremely characteristic). For instance, students are asked to rate the degree to which they "prefer complex to simple problems" or they "find satisfaction in deliberating for long hours."

Texts. Four texts were used that focused on two topics: Newton's first law of motion and Newton's third law of motion. The texts were adapted from a college-level physics textbook (Hewitt, 2002). We constructed two versions for each topic. One version followed a non- refutation format. The nonrefutation text was a shorter version of the original textbook section on Newton's laws of motion. It included an introduction, a definition of each law of motion, and several everyday examples and explanations related to each law. The other version followed a refutation format. The refutation text was also a shorter version of the original textbook section on Newton's laws of motion and included an introduction, a definition of each law, a few everyday examples and explanations related to each law, and refutations of certain misconceptions related to each law. Seven experts in science (four faculty members in science education and three graduate students in chemical engineering) reviewed and provided feedback for improving the initial versions of all four texts. The texts were finalized after accommodating the experts' suggestions. Texts were equated in word length (approximately 550 words each) and conventional readability indices. The average Flesch-Kincaid reading grade level was 8.3 .

\section{Procedure}

The participants were tested individually in a single session lasting approximately $1 \mathrm{~h} 40 \mathrm{~min}$. The participants were first administered the Force Concept Inventory. They were asked to read each question carefully and to select the correct answer to the best of their knowledge. Next, the participants were informed that they were going to read some texts and think aloud after every sentence (see Ericsson \& Simon, 1993, for a detailed description of the think-aloud methodology). The experimenter explained that thinking aloud was a process during which one is asked to state aloud his/her thoughts while reading a text. The participants were given the practice text and were asked to read each sentence on the card aloud and talk about their thoughts. They were also instructed to make sure they understood what they were reading. If the participant had difficulty talking aloud, the experimenter posed the following question: "What are you thinking right now?" When the practice text was completed, the participants were administered the first experimental text, and they were asked to read and think aloud after every sentence. They were also asked to make sure they understood what they were reading, because they would later be asked to recall the text. When the participants finished reading and thinking aloud, they were asked to complete a distractor task consisting of 10 math problems. After the distractor task, the participants were given a recall sheet and were asked to write down everything they could remember from the text they had read. The same procedure was followed with a second experimental text. The participants were administered one nonrefutation and one refutation text, in random order. Next, the participants completed the reading span test on a computer. Finally, the participants completed the Need for Cognition Scale.

\section{Scoring}

Prior knowledge tests. The Force Concept Inventory was scored dichotomously. Each correct response received a score of 1, whereas each incorrect response received a score of 0 . The observed total scores ranged from 3 to 30 (possible range $0-30$ ).

Think-aloud protocols. Students' responses during the thinkaloud procedure were transcribed. Two raters independently parsed and coded $25 \%$ of the protocols into clauses, and their agreement was reliably high for both parsing $(K=.90, p<.01)$ and coding $(K=$ $.87, p<.01)$. Disagreements were resolved through discussion, and the remaining protocols were divided among the two raters for them to parse and code them independently. Each clause was categorized on the basis of a coding scheme adapted from previous research (Kendeou \& van den Broek, 2005; Linderholm \& van den Broek, 2002; Pritchard, 1990). The coding scheme identified the cognitive processes in which readers engaged during reading. It consisted of the following eight categories (example responses are included in parentheses). Comprehension monitoring included responses that showed (1) readers' awareness of how much progress they were making, (2) any problems readers encountered, and (3) whether the readers understood or failed to understand (e.g., "This makes sense regarding inertia"). Associations included responses that showed 
that readers activated prior knowledge related or unrelated to the text material (e.g., "I have seen this done before, where the tablecloth is removed quickly and the dishes remain on the table"). Intrasentential connections included readers' attempts to develop an understanding of the current sentence by rereading or paraphrasing it (e.g., "All objects continue in a state of rest unless acted on by another force"). Intersentential connections included readers' attempts to relate the current text to other sentences in the text (e.g., "Continues is also an important word, in addition to change"). Correct inferences included correct explanatory, forward, and other inferences that were based on readers' prior knowledge and text (e.g., "So, if there is no change in forces, there will be no change in motion"). Incorrect inferences included incorrect explanatory, forward, and other inferences that were based on readers' prior knowledge and the text (e.g., "So, inertia is the force that changes an object's motion"). Conceptual change strategies included responses that showed that readers were engaging in conceptual change, such as experiencing cognitive conflict, responding to conflict, and contrasting information (e.g., "Greater mass certainly leads me to believe that the forces are more forceful in my mind, but apparently that is not true"). Responses that did not fall into any of the categories above were categorized as other (e.g., "We will see next").

Recall protocols. Students' written recall protocols were parsed into clauses. Each clause was matched to the text sentences according to a gist criterion. Two raters independently parsed and coded $25 \%$ of the recall protocols, and their agreement was reliably high, for both parsing $(K=.95, p<.01)$ and coding $(K=.90, p<.01)$. Disagreements were resolved through discussion, and the remaining protocols were divided among the two raters for them to parse and code independently.

\section{Results}

We performed preliminary analyses to explore whether the misconception and nonmisconception groups differed with regard to the individual difference measures. The results show that there was no significant difference between the reading span scores for the misconception $(M=38.3, S D=4.9)$ and nonmisconception $(M=37.5$, $S D=6.7)$ groups $[F(1,78)<1, p=.5]$. There was a significant difference, however, in the Need for Cognition score, with the misconception group scoring significantly lower $(M=65.8, S D=10.1)$ than the nonmisconception group $(M=72.9, S D=9.4)[F(1,78)=8.9, p=.004$, $\left.\eta^{2}=.10\right]$. To eliminate the possibility of a confound due to individual differences in the reading span and Need for Cognition scores, individuals' scores on these measures were used as covariates in all subsequent analyses. Also, because we had two texts, with different topics for each structure, we included in the analysis a variable reflecting the topic of the text.

\section{Online Comprehension Processes}

We conducted separate repeated measures ANOVAs, with prior knowledge (misconception, nonmisconception), text structure (refutation, nonrefutation), and text topic (Newton's first law, Newton's third law) as independent variables and frequency for each of the seven think-aloud response categories (in the two texts and across participants) as a dependent variable. Reading span and Need for Cognition variables were entered as covariates. The means for each response category are provided in Table 1 .

There were significant differences as a function of prior knowledge with respect to correct $[F(1,71)=13$, $\left.p=.001, \eta^{2}=.15\right]$ and incorrect $[F(1,71)=35.5, p=$ $\left..0001, \eta^{2}=.33\right]$ inferences. In particular, the misconception group generated significantly fewer correct inferences $(M=7.5)$ than did the nonmisconception group $(M=13.9)[t(71)=3.6, p<.001]$ and significantly more incorrect inferences $(M=3.8)$ than did the nonmisconception group $(M=0.5)[t(71)=5.9, p<.001]$. There were also significant prior knowledge differences with regard to conceptual change strategies $[F(1,71)=50.1$, $\left.p=.0001, \eta^{2}=.41\right]$. The misconception group generated significantly more conceptual change strategies $(M=$ $3.5)$ than did the nonmisconception group $(M=0.2)$ $[t(71)=7.1, p<.001]$. This main effect was qualified by an interaction between prior knowledge and text structure $\left[F(1,71)=50.8, p=.0001, \eta^{2}=.42\right]$. Post hoc comparisons showed that the misconception group included more conceptual change strategies than did the nonmisconception group, but only during their reading of the refutation text $[t(71)=4.5, p<.001]$.

Finally, there were no significant prior knowledge differences with respect to the remaining four types of processes: comprehension monitoring $[F(1,71)<1, p=.5]$, associations $[F(1,71)=2.1, p=.1]$, intrasentential connections $[F(1,71)<1, p=.5]$, and intersentential connections $[F(1,71)<1, p=.8]$. No other main effects or

Table 1

Mean Frequencies and Standard Deviations of the Types of Processes During the Think-Aloud Phase, As a Function of Prior Knowledge (Misconception, Nonmisconception) and Text Structure (Refutation, Nonrefutation) in Experiment 1

\begin{tabular}{|c|c|c|c|c|c|c|c|c|}
\hline \multirow[b]{3}{*}{ Processes } & \multicolumn{4}{|c|}{ Misconception } & \multicolumn{4}{|c|}{ Nonmisconception } \\
\hline & \multicolumn{2}{|c|}{ Refutation } & \multicolumn{2}{|c|}{$\overline{\text { Nonrefutation }}$} & \multicolumn{2}{|c|}{ Refutation } & \multicolumn{2}{|c|}{ Nonrefutation } \\
\hline & $M$ & $\overline{S D}$ & $M$ & $\overline{S D}$ & $M$ & $\overline{S D}$ & $M$ & $\overline{S D}$ \\
\hline Comprehension monitoring & 15.3 & 9.6 & 15.1 & 8.8 & 14.1 & 8.3 & 14.2 & 7.8 \\
\hline Associations & 12.9 & 9.1 & 17.9 & 8.9 & 13.6 & 7.8 & 16.5 & 8.1 \\
\hline Intrasentential connections & 11.1 & 7.7 & 13.7 & 7.3 & 9.3 & 8.3 & 10.9 & 11.4 \\
\hline Intersentential connections & 3.2 & 2.5 & 4.0 & 2.4 & 4.0 & 4.0 & 3.2 & 2.7 \\
\hline Correct inferences* & 10.0 & 7.3 & 5.4 & 4.2 & 16.3 & 11.0 & 10.9 & 7.6 \\
\hline Incorrect inferences* & 3.9 & 3.4 & 4.0 & 4.0 & 0.5 & 1.5 & 0.3 & 0.7 \\
\hline Conceptual change strategies ${ }^{* \dagger}$ & 6.2 & 4.6 & 0.9 & 1.5 & 0.1 & 0.4 & 0.1 & 0.6 \\
\hline
\end{tabular}

Note-The means are adjusted with reference to the covariates. ${ }^{*} p<.05$, main effect of prior knowledge. $\quad t p<.05$, interaction effect of prior knowledge and text structure. 
interactions were significant for the seven processes identified during thinking aloud (all $p \mathrm{~s}>.2$ ).

\section{Offline Recall}

We conducted a repeated measures ANOVA, with prior knowledge (misconception, nonmisconception), text structure (refutation, nonrefutation), and text topic (Newton's first law, Newton's third law) as independent variables, and frequency of recall for the texts as the dependent variable. Reading span and Need for Cognition variables were entered as covariates. This analysis revealed a significant effect of prior knowledge $\left[F(1,74)=5.8, p=.02, \eta^{2}=\right.$ $.08]$. As can be seen in Table 2 , readers with misconceptions recalled less textual information than did readers with no misconceptions $[t(74)=2.4, p<.05]$, regardless of structure or topic. No other main effects or interactions were significant (all $p \mathrm{~s}>.2$ ).

\section{Connecting Online Processes and Offline Recall}

We explored whether the patterns of think-aloud responses during reading were related to subsequent text recall. We performed a hierarchical multiple regression analysis for each group (misconception, nonmisconception), with the proportion of sentences recalled as the dependent variable and, as independent variables, a dichotomous text structure variable (refutation, nonrefutation) in the first step and the number of each of the seven types of think-aloud responses (associations, comprehension monitoring, intrasentential connections, intersentential connections, correct inferences, incorrect inferences, and conceptual change) in the second step. For the misconception group, the regression model for text structure (first step) did not account for any significant variance in text recall $[F(1,75)=2.2, p>$.7]. The regression model for the think-aloud responses (second step), however, accounted for a significant $18 \%$ of the variance in text recall $[F(7,68)=2.2, p=.04]$. Specifically, the number of correct inferences that occurred during the think-aloud phase strongly predicted the proportion of sentences recalled $[\beta=.37, t(68)=2.4, p<.05]$ : The greater the number of correct inferences an individual produced, the more that individual recalled. None of the other six think-aloud predictors were significant (all $p \mathrm{~s}>.2$ ). For the nonmisconception group, neither regression model (for text structure and think-aloud responses, respectively) accounted for any significant variance in text recall (both $p \mathrm{~s}>.4$ ).

\section{Discussion}

The online results show that the processes in which readers with misconceptions and readers without misconceptions engage during their reading of scientific texts are similar in some respects, yet different in others. In particular, readers with misconceptions engage in more conceptual change strategies than do readers without misconceptions during the reading of a refutation text, but not during reading of a nonrefutation text. In addition, in both text structures, readers with misconceptions generate more incorrect and fewer correct inferences than do readers without misconceptions.
Table 2

Average Number of Statements Recalled As a Function of Prior Knowledge (Misconception, Nonmisconception) and Text Structure (Refutation, Nonrefutation) in Experiments 1 and 2

\begin{tabular}{llllll}
\hline & \multicolumn{2}{c}{ Misconception } & & \multicolumn{2}{c}{ Nonmisconception } \\
\cline { 2 - 3 } \cline { 6 - 6 } Text Structure & $M$ & $S D$ & & $M$ & $S D$ \\
\hline Experiment 1 & & & & \\
$\quad$ Refutation* & 11.5 & 3.4 & & 14.0 & 4.6 \\
$\quad$ Nonrefutation* & 11.0 & 4.5 & & 13.5 & 5.2 \\
Experiment 2 & & & & \\
$\quad$ Refutation* & 14.0 & 3.5 & & 16.2 & 3.7 \\
$\quad$ Nonrefutation* & 11.6 & 3.6 & & 14.2 & 4.7 \\
\hline
\end{tabular}

Note-The means are adjusted with reference to the covariates. $\quad{ }^{*} p<.05$, main effect of prior knowledge.

The offline results show that readers' memory for both refutation and nonrefutation texts is affected by prior knowledge. These results are consistent with those obtained in prior research demonstrating that misconceptions have an intrusive effect on students' memory representations of text (Guzzetti et al., 1993; Kendeou \& van den Broek, 2005).

The findings also show that the online processes were reflected in memory once reading was completed. In particular, for readers with misconceptions, the number of correct inferences generated during the think-aloud phase was associated with higher recall for the text. This was not the case for readers without misconceptions. These findings suggest that the two groups of readers differentially benefited from constructing correct inferences during reading. ${ }^{1}$ One possible explanation for this interesting finding relates to the function of correct inferences. It is conceivable that the generation of correct inferences increased the compatibility of readers' prior knowledge and the text. So, in the case of readers with misconceptions, more correct inferences increased the coherence between their knowledge and the text, which in turn increased their text recall. For readers without misconceptions, the existing coherence between readers' prior knowledge and the text was already at a high level, so correct inferences had no additional benefit in that direction. An alternative explanation relates to the presence of a mediating variable - that of conceptual change processes. It is likely that the more conceptual change processes readers produced, the more correct inferences they produced, which in turn led to better recall. In this case, generating correct inference alone (as in the case of readers without misconceptions) made no contribution to readers' text recall. We explored this possibility in our data, and bivariate correlations showed that, indeed, in the misconception group, correct inferences and conceptual change processes were significantly related $(r=.45, p<.01)$, correct inferences and recall were also significantly related $(r=$ $.42, p<.01)$, but conceptual change processes and recall were not directly related $(r=-.04, p>.8)$. In the nonmisconception group, there were no significant relations between any of these variables. Although further research is needed to investigate these issues, these tentative explanations can provide some insight into why correct inferences contributed to better recall for readers with misconceptions and did not for readers without misconceptions. 
In this experiment, we used a think-aloud methodology to investigate online processing and to obtain a variety of students' responses. Think-aloud procedures provide a rich source of data, but they also have several limitations. One limitation is that this methodology reveals only a subset of cognitive processes during reading - namely, those of which readers are aware. However, readers also engage in processes of which they are not aware. For instance, when readers encounter information in a text that is inconsistent with information in the preceding text, they slow down without necessarily being aware of doing so (Albrecht \& O'Brien, 1993; Myers, O'Brien, Albrecht, $\&$ Mason, 1994). A second limitation of the think-aloud methodology is that it may alter readers' spontaneous processing of the text, resulting in changes in comprehension (Fletcher, 1986). Because of these limitations, it is important to obtain converging evidence by using a less intrusive and more automatic measure (Magliano \& Graesser, 1991). Thus, in Experiment 2, we obtained reading time measures for the text sentences. Reading times at the word, sentence, and text levels have been used extensively in psychological research as an indicator of processing (Lorch \& van den Broek, 1997; O’Brien, 1995; Rapp, Gerrig, \& Prentice, 2001; Zwaan \& Singer, 2003).

\section{EXPERIMENT 2 Reading Times Methodology}

The objective of Experiment 2 was to investigate the interaction between prior knowledge and text structure online, using a reading time methodology. In this experiment, participants with and without misconceptions were asked to read a refutation and a nonrefutation scientific text on the computer, one sentence at a time. We investigated reading times for sentences that were relevant for understanding the scientific laws described in each textnamely, the sentences stating Newton's laws and those that explained these laws. As in Experiment 1, participants were also asked to recall the texts they read and to complete the measures of working memory capacity and the Need for Cognition Scale.

We made several predictions with respect to the outcomes of this experiment. First, as in Experiment 1, we expected that the interaction between readers' prior knowledge and text structure would affect readers' online comprehension processes. In particular, given the findings of Experiment 1, we expected that readers with misconceptions would spend more time reading sentences that contradicted their prior knowledge (Newton's laws and explanations) than would readers without misconceptions. Given the interaction effect of text structure and prior knowledge observed in Experiment 1, this effect would most obviously obtain when participants read refutation text structures. Second, we expected to replicate the offline results obtained in Experiment 1 - that readers with misconceptions would remember less information from the texts than would readers without misconceptions. This result would suggest that the think-aloud methodology in Experiment 1 did not alter readers' normal processing.
Finally, we expected a convergence between the online results obtained in Experiment 1 and those obtained in Experiment 2, with the think-aloud processes revealed during reading by one group of readers predicting reading times of another group of readers during silent reading. Validating the think-aloud data with independent behavioral measures such as reading times helps strengthen any conclusions that might be drawn from either data set alone (Fletcher, 1986; Magliano \& Graesser, 1991; Magliano et al., 1999).

\section{Method}

\section{Participants}

A total of 69 University of Minnesota undergraduates enrolled in introductory psychology or physics courses participated in this study. The participants received extra course credit or a gift certificate for participating. The data of 9 students were eliminated from the study because they were nonnative English speakers. Of the remaining participants, 30 were female and 30 were male, with an age range of 18-32 years. Thirty participants ( 20 female, 10 male) made up the misconception group (students who scored $60 \%$ or less on the Force Concept Inventory). The remaining 30 participants (who scored above $60 \%$ on the Force Concept Inventory) made up the nonmisconception group (10 female, 20 male).

\section{Materials \\ The materials were the same as those in Experiment 1.}

\section{Procedure}

The participants were seen individually in a single session. The session lasted approximately $1 \mathrm{~h} 10 \mathrm{~min}$. The procedure was similar to that of Experiment 1, with one difference: Instead of reading the two texts using the think-aloud methodology, the participants simply read the texts silently on a computer screen one sentence at a time, at their own speed. The participants advanced from one sentence to the next by pressing the space bar.

\section{Scoring}

Individual difference tests. The Force Concept Inventory, the Need for Cognition Scale, and the reading span test were scored as in Experiment 1.

Recall protocols. Students' written recall protocols were parsed and coded as in Experiment 1. Two raters independently parsed and coded $25 \%$ of the recall protocols, and their agreement was reliably high, for both parsing $(K=.98, p<.01)$ and coding $(K=.94$, $p<.01)$. Disagreements were resolved through discussion, and the remaining protocols were divided among the two raters for them to parse and code independently.

\section{Results}

We performed preliminary analyses to explore whether misconception and nonmisconception groups differed with regard to the individual difference measures. The analysis showed that there were no significant differences in reading span $[F(1,56)<1, p=.9]$ or in Need for Cognition $[F(1,56)=2.2, p=.1]$ scores between misconception (reading span, $M=39.2, S D=3.9$; Need for Cognition, $M=65.3, S D=12.9)$ and nonmisconception (reading span, $M=38.9, S D=4.3$; Need for Cognition, $M=69.3, S D=7.5$ ) groups. As in Experiment 1, the reading span and Need for Cognition scores for each individual were used as covariates in all subsequent analyses, to eliminate possible confounding of text structure and prior knowledge effects with these factors. Also, because 
we had two texts, with different topics for each structure, we included in the analysis a variable reflecting the topic of the text.

\section{Online Reading Times}

We performed an analysis on the reading times per syllable (to adjust for variability in sentence length) for target Newton's laws and explanation sentences from each text. Newton's law sentences explicitly stated the correct information about Newton's laws of motion, whereas explanation sentences provided evidence for the refutations in the refutation texts and for the supporting examples in the nonrefutation texts (see Appendix for examples). Explanation sentences differed in content across topics and text structures, so they may be compared only with caution. We removed outlying reading time data using Tukey's (1977) hinge criterion. This eliminated approximately $5.34 \%$ of the data. We conducted separate repeated measures ANOVAs, with prior knowledge (misconception, nonmisconception), text structure (refutation, nonrefutation), and text topic (Newton's first law, Newton's third law) as the independent variables and average reading times for each selected category of target sentences as the dependent variables. Reading span and Need for Cognition variables were entered as covariates. The means are provided in Table 3 .

For reading times of Newton's law sentences, the analysis revealed a significant effect of text structure $\left[F(1,44)=16.5, p=.001, \eta^{2}=.27\right]$. Post hoc analyses showed that readers read Newton's law sentences faster in the refutation $(M=201)$ than in the nonrefutation $(M=$ 295) $[t(44)=-4.1, p<.01]$ texts. This main effect was qualified by an interaction between text structure and prior knowledge $\left[F(1,44)=8.3, p=.006, \eta^{2}=.16\right]$. Post hoc analyses showed that readers with misconceptions spent more time reading the law sentences than did readers without misconceptions in the refutation texts $[t(44)=4.3, p<.01]$, but not in the nonrefutation texts $[t(44)<1, p>.05]$.

For the explanation sentences, the analysis also revealed a significant effect of text structure $[F(1,52)=11.8, p=$ $\left..001, \eta^{2}=.18\right]$. Post hoc analyses showed that readers read the explanation sentences faster in the refutation $(M=206)$ than in the nonrefutation $(M=253)[t(52)=$ $-3.4, p<.01]$ texts. No other main effects or interactions were significant (all $p \mathrm{~s}>.3$ ).

\section{Offline Recall}

We conducted a repeated measures ANOVA, with prior knowledge (misconception, nonmisconception), text structure (refutation, nonrefutation), and text topic (Newton's first law, Newton's third law) as the independent variables and frequency of recall for the two texts as the dependent variable. Reading span and Need for Cognition variables were entered as covariates. This analysis revealed a significant effect of prior knowledge $[F(1,54)=6.6, p=.01$, $\left.\eta^{2}=.11\right]$. As can be seen in Table 2, readers with misconceptions recalled less text information than did readers with no misconceptions $[t(54)=2.5, p<.05]$. No other main effects or interactions were significant (all $p s>.2$ ).

\section{Connecting Think-Aloud Responses (Experiment 1) and Reading Times}

(Experiment 2)

We assessed the relation between the thoughts produced during the think-aloud phase in Experiment 1 and the sentence reading times in Experiment 2, using the procedures suggested by Magliano and colleagues (Magliano \& Graesser, 1991; Magliano et al., 1999). To do so, we tested whether the frequency of think-aloud processes produced in Experiment 1 predicted the reading times during silent reading obtained in Experiment 2. Following Magliano et al., we performed multiple regression analysis in which the predictor variables consisted of the average frequency of each of the seven types of cognitive processes for each sentence produced in Experiment 1, and the dependent variable consisted of the average reading time for each sentence recorded in Experiment 2. We also included three variables to control for (1) the number of syllables in each sentence, (2) text structure (refutation, nonrefutation), and (3) prior knowledge (misconception, nonmisconception). As we have seen from the previous analyses, text structure and prior knowledge significantly influence think-aloud responses and reading times, so these were included as dichotomous variables.

The regression model accounted for a significant $74 \%$ of the variance in sentence reading times $[F(10,271)=76.9$, $p=.001]$. Two factors were responsible for this effect. Sentence reading times increased as a function of the number of syllables in a sentence $[\beta=.84, t(271)=26, p<$ $.001]$ and as a function of the number of associations that occurred during the think-aloud phase $[\beta=.07, t(271)=$ $2.3, p<.05]:$ The longer the sentence and the more asso-

Table 3

Average Reading Times (Per Syllable) of Target Sentences in Experiment 2

As a Function of Prior Knowledge (Misconception, Nonmisconception) and Text Structure (Refutation, Nonrefutation)

\begin{tabular}{|c|c|c|c|c|c|c|c|c|}
\hline \multirow[b]{3}{*}{ Sentences } & \multicolumn{4}{|c|}{ Misconception } & \multicolumn{4}{|c|}{ Nonmisconception } \\
\hline & \multicolumn{2}{|c|}{ Refutation } & \multicolumn{2}{|c|}{ Nonrefutation } & \multicolumn{2}{|c|}{ Refutation } & \multicolumn{2}{|c|}{ Nonrefutation } \\
\hline & $M$ & $S D$ & $M$ & $S D$ & $M$ & $S$ & $M$ & $S D$ \\
\hline Laws $^{* \dagger}$ & 252 & 84 & 280 & 138 & 148 & 84 & 310 & 138 \\
\hline Explanations* & 217 & 72 & 254 & 82 & 195 & 72 & 253 & 86 \\
\hline
\end{tabular}

Note-The means are adjusted with reference to the covariates. ${ }^{*} p<.05$, main effect of text structure. $t_{p}<<.05$, interaction effect of prior knowledge and text structure. 
ciations a sentence elicited, the longer the reading time. No other predictors were significant (all $p \mathrm{~s}>.2$ ).

\section{Discussion}

The online reading times suggest that when readers with misconceptions read refutation texts, they detect the contradiction between their faulty prior beliefs and the information presented in the text. Newton's law sentences were read more slowly by readers with misconceptions about the laws than by readers who had no such misconceptions, but only when these sentences were included in a refutation text. With respect to the explanation sentences, the results showed that there were no differences in the reading times of the two groups. Although we expected that readers with misconceptions would spend more time reading the explanation sentences in the refutation than in the nonrefutation texts because the explanations would assist them in reconciling the inconsistent information, the lack of differences may be due in part to the fact that explanations differed in content across texts; hence, these findings should be interpreted with caution.

Interestingly, all readers read the target sentences faster in the refutation texts than in the nonrefutation texts. One possibility is that refutation texts were on average easier to read than nonrefutation texts. All texts, however, were controlled with respect to conventional readability measures, including word length, grade level, and ease of reading. So there is no indication that the refutation texts were in fact easier. Another possibility is that refutation texts result in a more coherent representation because they elicit an explicit integration between readers' potential background knowledge and the to-be-learned material and, hence, greater reading speed. This remains an empirical question to be addressed in the future. It is important to note that even though we observed a text structure effect on reading times in this experiment, no corresponding effect was observed on conceptual change processes in Experiment 1. Given that readers without misconceptions did not engage in conceptual change processes in Experiment 1, the latter finding most likely reflects a floor effect.

The offline findings demonstrate that readers' memory for both text structures (refutation, nonrefutation) was a function of their prior knowledge. Readers with misconceptions recalled less textual information than did readers with no misconceptions across the two text structures. These findings are consistent with those obtained in Experiment 1 . Thus, they provide convergent evidence for our conclusions about online processes and, moreover, suggest that the think-aloud methodology did not alter readers' normal reading.

The findings also demonstrate that there is a direct connection between the online processes in the two experiments. Indeed, the think-aloud responses obtained in Experiment 1 predicted reading times for sentences in Experiment 2. Specifically, the number of associations readers generated significantly predicted reading times of sentences during silent reading. Note that in the present study, we defined associations as responses that reflected activation of prior knowledge, including knowledge that was related to the text material. The observation that such activation requires additional time is consistent with current theories of reading comprehension, according to which readers routinely activate prior knowledge from memory when it is required for comprehension, through memorybased and constructionist processes (Graesser, Singer, \& Trabasso, 1994; McKoon \& Ratcliff, 1992; Myers \& O'Brien, 1998). Whereas activation of prior knowledge through memory-based processes may occur without a processing cost (van den Broek et al., 2005), activation of prior knowledge using strategy-based processes - the ones likely to be reflected in think-aloud protocolswould require additional time.

\section{GENERAL DISCUSSION}

The aim of this set of experiments was to investigate the effects of text structure and readers' prior knowledgeand their possible interaction - on the comprehension processes that take place during reading of scientific texts. We focused particularly on the processing of refutation and nonrefutation scientific texts by readers with and without misconceptions related to the topics of the text. We also took into account readers' individual differences in working memory capacity and Need for Cognition scores as possible factors that might influence processing in reading situations of this nature.

The online results provide direct evidence for the interactive effects of readers' prior knowledge and text structure during reading. Readers with misconceptions engaged in different processes than did readers without misconceptions, but only during reading of text with the refutation structure. Specifically, readers with misconceptions engaged in conceptual change strategies more than did readers without misconceptions when reading a refutation text (Experiment 1). These conceptual change strategies included statements indicating that readers were experiencing conflict, responding to conflict, and contrasting information. Furthermore, readers with misconceptions spent more time reading sentences describing Newton's laws that directly contradicted their misconceptions about those laws than did readers without misconceptions, but only when those sentences were included in a refutation text (Experiment 2). Thus, our findings provide evidence that readers adjusted their processing of the text when prior knowledge conflicted with textual information during comprehension of the refutation texts. This pattern of results was not present when readers - with and without misconceptions - read the nonrefutation texts.

With regard to other comprehension processes (i.e., associations, establishing connections within and across sentences, generating explanatory, predictive, and other inferences, and comprehension monitoring), the results indicate that the frequency of these processes was not affected by readers' prior knowledge or by text structure but that the content of some of them was (Experiment 1). In particular, readers with misconceptions generated more incorrect inferences and fewer correct inferences than did readers without misconceptions. 
These findings add to our understanding of the role of text structure and readers' misconceptions during online text processing of scientific texts by demonstrating that the actual reading processes themselves, as they unfold during reading of the text, are influenced by both misconceptions and text structure. On the one hand, readers with misconceptions proceed through nonrefutation texts at the same rate and with the same types of processes as do readers without misconceptions. When the text calls for it, the readers with misconceptions activate and integrate their background knowledge with the textual information as frequently as do readers without misconceptions (see also Hannon \& Daneman, 2001; Kintsch, 1988). But the content of knowledge-based inferences (e.g., explanatory, predictive, and other inferences) reflects their misconceptions, resulting in more incorrect inferences and fewer correct ones. On the other hand, readers with misconceptions proceed through refutation texts at a slower rate and rely on different processes than do readers without misconceptions. When the text contradicts their prior knowledge, they spend more time reading and they engage in conceptual change strategies.

Previous research in reading and science education has suggested that refutation texts are effective means of conceptual change learning (Guzzetti et al., 1993). This conclusion stemmed primarily from results obtained with offline tasks, in which students' learning was measured using application questions or problem-solving activities. Such offline measures provide limited insight into what happens during reading, however, and restrict claims with respect to readers' cognitive processing. The results of the present study allow us to go beyond these findings and consider how the actual reading processes themselves unfold. They provide evidence that during online reading of refutation text, readers coactivate and integrate prior knowledge and text information, which, in turn, allows them to detect the inconsistency between their knowledge and the text. Detecting the inconsistency enables readers to engage in additional processing in an attempt to establish coherence (Glenberg, Wilkinson, \& Epstein, 1982; Graesser et al., 1994; McNamara \& Kintsch, 1996) or reconcile the inconsistent information (e.g., Hakala \& O'Brien, 1995; Linderholm, Virtue, Tzeng, \& van den Broek, 2004). The present findings indicate that refutation texts help readers engage in conceptual change learning by eliciting coactivation, thereby facilitating inconsistency detection and the establishment of coherence.

With regard to the offline text representation, readers' misconceptions affected their memory for the text in both experiments in the same way: Regardless of text structure, readers with misconceptions remembered less information than did readers without misconceptions. Thus, the conceptual change processes elicited by refutation texts in readers with misconceptions do not inevitably result in successful revision of their incorrect ideas. This may reflect the fact that successful revision is a function of many factors, including the sufficiency of an alternative explanation, the plausibility of the new scientific information, and readers' commitment to their preexisting beliefs (Dole, 2000; Posner, Strike, Hewson, \& Gertzog, 1982). Alternatively, it is possible that readers with misconceptions successfully revised their incorrect ideas but that the recall measure was not sensitive enough to capture this effect. Evidence for revising prior knowledge is most likely to be reflected on tasks that assess readers' learning from text (as opposed to memory for the text) - that is, on tasks that require readers to apply the acquired knowledge in a new situation (Guzzetti et al., 1993; Kintsch, 1998).

The described results are independent of individual differences in working memory and Need for Cognition. On average, readers with and readers without misconceptions did not differ in their working memory capacity in either experiment. In Experiment 1, readers with misconceptions on average had lower Need for Cognition scores than did their counterparts without misconceptions, suggesting that readers with misconceptions may maintain lower standards for coherence (van den Broek, Risden, \& Husebye-Hartmann, 1995). Although the latter difference was not repeated in Experiment 2, we included both variables as covariates in all analyses, to eliminate any possible confounds. In none of the analyses did either the working memory capacity or the Need for Cognition score account for a significant portion of the variance. Thus, the observed effects of readers' prior knowledge and text structure are not the result of individual differences in working memory capacity or Need for Cognition.

Different types of online data were obtained in the two experiments. Although these data were collected from different participants, comparison of the two types of information about online processes may suggest interesting connections $^{2}$ (Fletcher, 1986; Magliano \& Graesser, 1991; Magliano et al., 1999). In particular, in light of the focus of the present study on the role of background knowledge, we explored whether the activation of such information was related to the speed of reading. Indeed, the number of associations that participants produced in response to different sentences (Experiment 1) significantly predicted reading times for the same sentences (Experiment 2). No other think-aloud process significantly predicted reading times, including knowledge-based inferences. The finding that associations did but knowledge-based inferences did not predict processing times suggests that, consistent with current theories of reading comprehension, activation of prior knowledge may occur with (Graesser et al., 1994) and without (McKoon \& Ratcliff, 1992; Myers \& O’Brien, 1998) a processing cost.

In conclusion, the findings of this study contribute to our theoretical understanding of the effects that reader characteristics, such as background knowledge, and text properties have on readers' cognitive processes during comprehension. This understanding is particularly important in the context of comprehension of scientific texts, because aspects of the reading situation besides the content itself - such as readers' individual differences in domain knowledge, strategies, attention, and motivation, as well as text properties such as structure - strongly codetermine the likelihood of acquisition of the content in such texts. 


\section{AUTHOR NOTE}

This project was supported by a Robert and Corrie Beck Fellowship from the College of Education and Human Development at the University of Minnesota and by a dissertation research grant from the Graduate School at the University of Minnesota to P. K., by a Golestan and Lorentz fellowship at the Netherlands Institute for Advanced Study in the Humanities and Social Sciences to P. v. d. B., and by the Center for Cognitive Sciences at the University of Minnesota through Grant HD-07151 from the National Institute of Child Health and Human Development. We thank Joe Magliano, David N. Rapp, and two anonymous reviewers for their advice and comments on the manuscript. Correspondence concerning this article should be addressed to P. Kendeou, Faculty of Education, McGill University, 3700 McTavish Avenue, Montreal, QC, H3A 1Y2 Canada (e-mail: panayiota.kendeou@mcgill.ca).

\section{REFERENCES}

AfFlerbach, P. (2002). Verbal reports and protocol analysis. In M. L. Kamil, P. B. Mosenthal, P. D. Pearson, \& R. Barr (Eds.), Handbook of reading research (Vol. III, pp. 87-103). Mahwah, NJ: Erlbaum.

Albrecht, J. E., \& O’Brien, E. J. (1993). Updating a mental model: Maintaining both local and global coherence. Journal of Experimental Psychology: Learning, Memory, \& Cognition, 19, 1061-1069.

Alvermann, D. E., \& Hague, S. A. (1989). Comprehension of counterintuitive science text: Effects of prior knowledge and text structure. Journal of Educational Research, 82, 197-202.

Alvermann, D. E., \& Hynd, C. (1989). Effects of prior knowledge activation modes and text structure on nonscience majors' comprehension of physics. Journal of Educational Research, 83, 97-102.

Alvermann, D. E., Smith, L. C., \& Readence, J. E. (1985). Prior knowledge activation and the comprehension of compatible and incompatible text. Reading Research Quarterly, 20, 420-436.

Baddeley, A., \& Hitch, G. (1974). Working memory. Psychology of Learning \& Motivation, 8, 47-90.

Bartlett, F. C. (1932). Remembering: A study in experimental and social psychology. Cambridge: Cambridge University Press.

Budd, D., Whitney, P., \& TuRley, K. J. (1995). Individual differences in working memory strategies for reading expository text. Memory \& Cognition, 23, 735-748.

CaCioppo, J. T., \& Petty, R. E. (1982). The need for cognition. Journal of Personality \& Social Psychology, 42, 116-131.

Chambliss, M. J. (2002). The characteristics of well-designed science textbooks. In J. Otero, J. A. Leon, \& A. C. Graesser (Eds.), The psychology of science text comprehension (pp. 1-15). Mahwah, NJ: Erlbaum.

CHI, M. T. H. (1978). Knowledge structures and memory. In R. S. Siegler (Ed.), Children's thinking: What develops? (pp. 73-96). Hillsdale, NJ: Erlbaum.

Chi, M. T. H., Feltovich, P. J., \& Glaser, R. (1981). Categorization and representation of physics problems by experts and novices. Cognitive Science, 5, 121-152.

Chiesi, H. L., Spilich, G. J., \& Voss, J. F. (1979). Acquisition of domain-related information in relation to high and low domain knowledge. Journal of Verbal Learning \& Verbal Behavior, 18, 257-273.

Coté, N., \& Goldman, S. R. (1999). Building representations of informational text: Evidence from children's think-aloud protocols. In H. van Oostendorp \& S. R. Goldman (Eds.), The construction of mental representations during reading (pp. 169-193). Mahwah, NJ: Erlbaum.

Diakidoy, I. N., \& Kendeou, P. (2001). Facilitating conceptual change in astronomy: A comparison of the effectiveness of two instructional approaches. Learning \& Instruction, 11, 1-20.

Diakidoy, I. N., Kendeou, P., \& IoAnnides, C. (2003). Reading about energy: The effects of text structure in science learning and conceptual change. Contemporary Educational Psychology, 28, 335-356.

Dochy, F., Segers, M., \& Buehl, M. M. (1999). The relation between assessment practices and outcomes of studies: The case of research on prior knowledge. Review of Educational Research, 69, 145-186.

Dole, J. A. (2000). Readers, texts and conceptual change learning. Reading \& Writing Quarterly, 16, 99-118.

ENGLE, R. W. (2002). Working memory capacity as executive attention. Current Directions in Psychological Science, 11, 19-23.
Engle, R. W., \& Conway, A. R. A. (1998). Working memory and comprehension. In R. H. Logie \& K. J. Gilhooly (Eds.), Working memory and thinking (pp. 67-91). East Sussex, U.K.: Psychology Press.

Ericsson, K. A., \& Simon, H. A. (1993). Protocol analysis: Verbal reports as data. Cambridge, MA: MIT Press.

FLetcher, C. R. (1986). Strategies for the allocation of short-term memory during comprehension. Journal of Memory \& Language, 25, 43-58.

Glenberg, A. M., Wilkinson, A. C., \& Epstein, W. (1982). The illusion of knowing: Failure in the self-assessment of comprehension. Memory \& Cognition, 10, 597-602.

Goldman, S. R., \& Bisanz, G. L. (2002). Toward a functional analysis of scientific genres: Implications for understanding and learning processes. In J. Otero, J. A. Leon, \& A. C. Graesser (Eds.), The psychology of science text comprehension (pp. 19-50). Mahwah, NJ: Erlbaum.

Goldman, S. R., \& Varma, S. (1995). CAPing the constructionintegration model of discourse comprehension. In C. A. Weaver, S. Mannes, \& C. R. Fletcher (Eds.), Discourse comprehension: Essays in honor of Walter Kintsch (pp. 337-358). Hillsdale, NJ: Erlbaum.

Graesser, A. C., Leon, J. A., \& Otero, J. (2002). Introduction to the psychology of science text comprehension. In J. Otero, J. A. Leon, \& A. C. Graesser (Eds.), The psychology of science text comprehension (pp. 1-15). Mahwah, NJ: Erlbaum.

Graesser, A., Singer, M., \& Trabasso, T. (1994). Constructing inferences during narrative comprehension. Psychological Review, 101, 371-395.

Guthrie, J. T., McGough, K., Bennett, L., \& Rice, M. E. (1996). Concept-oriented reading instruction: An integrated curriculum to develop motivations and strategies for reading. In L. Baker, P. Afflerbach, \& D. Reinking (Eds.), Developing engaged readers in school and home communities (pp. 165-190). Hillsdale, NJ: Erlbaum.

Guzzetti, B. J., Snyder, T. E., Glass, G. V., \& Gamas, W. S. (1993). Promoting conceptual change in science: A comparative meta-analysis of instructional interventions from reading education and science education. Reading Research Quarterly, 28, 117-159.

Hakala, C. M., \& O'Brien, E. J. (1995). Strategies for resolving coherence breaks in reading. Discourse Processes, 20, 167-185.

Hannon, B., \& Daneman, M. (2001). A new tool for measuring and understanding individual differences in the component processes of reading comprehension. Journal of Educational Psychology, 93, 103128.

Hestenes, D., \& Halloun, I. (1995). Interpreting the Force Concept Inventory. The Physics Teacher, 33, 502-506.

Hestenes, D., Wells, M., \& Swackhamer, G. (1992). Force Concept Inventory. The Physics Teacher, 30, 141-158.

Hewitt, P. G. (2002). Touch this! Conceptual physics for everyone. Glenview, IL: Pearson Education.

Jenkins, J. J. (1979). Four points to remember: A tetrahedral model of memory experiments. In L. S. Cermak \& F. I. M. Craik (Eds.), Levels of processing in human memory (pp. 429-446). Hillsdale, NJ: Erlbaum.

KaRdash, C. M., \& Scholes, R. J. (1996). Effects of pre-existing beliefs, epistemological beliefs, and need for cognition on interpretation of controversial issues. Journal of Educational Psychology, 88, 260-271.

Kendeou, P., Rapp, D. N., \& van den Broek, P. (2004). The influence of readers' prior knowledge on text comprehension and learning from text. In R. Nata (Ed.), Progress in education (Vol. 13, pp. 189-209). New York: Nova Science.

Kendeou, P., \& VAN DEN Broek, P. (2005). The effects of readers' misconceptions on comprehension of scientific text. Journal of Educational Psychology, 97, 235-245.

Kintsch, W. (1988). The role of knowledge in discourse comprehension: A construction-integration model. Psychological Review, 95, 163-182.

KInTSCH, W. (1998). Comprehension: A paradigm for cognition. Cambridge: Cambridge University Press.

Langston, M. C., \& Trabasso, T. (1999). Modeling causal integration and availability of information during comprehension of narrative texts. In H. van Oostendorp \& S. R. Goldman (Eds.), The construction of mental representations during reading (pp. 29-69). Mahwah, NJ: Erlbaum. 
Linderholm, T., \& VAN DEN Broek, P. (2002). The effects of reading purpose and working memory capacity on the processing of expository text. Journal of Educational Psychology, 94, 778-784.

Linderholm, T., Virtue, S., Tzeng, Y., \& VAN DEN Broek, P. (2004). Fluctuations in the availability of information during reading: Capturing cognitive processes using the Landscape Model. Discourse Processes, 37, 165-186.

Lipson, M. Y. (1982). Learning new information from text: The role of prior knowledge and reading ability. Journal of Reading Behavior 14, 243-261.

LORCH, R. F., JR., \& VAN DEN BROEK, P. (1997). Understanding reading comprehension: Current and future contributions to cognitive science. Contemporary Educational Psychology, 22, 213-246.

Magliano, J. P., \& Graesser, A. C. (1991). A three-pronged method for studying inference generation in literary text. Poetics, 20, 193 232.

Magliano, J. P., \& Millis, K. K. (2003). Assessing reading skill with a think-aloud procedure and latent semantic analysis. Cognition \& Instruction, 21, 251-283.

Magliano, J. P., Trabasso, T., \& Graesser, A. C. (1999). Strategic processes during comprehension. Journal of Educational Psychology, 91, 615-629.

Maria, K., \& MacGinitie, W. (1987). Learning from texts that refute the reader's prior knowledge. Reading Research \& Instruction, 26 , 222-238.

McKoon, G., \& Ratcliff, R. (1992). Inference during reading. Psychological Review, 99, 440-466.

McNamara, D. S. (2001). Reading both high-coherence and lowcoherence texts: Effects of text sequence and prior knowledge. Canadian Journal of Experimental Psychology, 55, 51-62.

McNamara, D. S., \& Kintsch, W. (1996). Learning from texts: Effects of prior knowledge and text coherence. Discourse Processes, 22, 247-288.

McNamara, D. S., Kintsch, E., Songer, N. B., \& Kintsch, W. (1996). Are good texts always better? Text coherence, background knowledge, and levels of understanding in learning from text. Cognition \& Instruction, 14, 1-43.

Means, M. L., \& Voss, J. F. (1985). Star Wars: A developmental study of expert and novice knowledge structures. Journal of Memory \& Language, 24, 746-757.

MEYER, B. J. F. (1975). The organization of prose and its effects on memory. Amsterdam: North-Holland.

MEYeR, B. J. F. (1999). Importance of text structure in everyday reading. In A. Ram \& K. Moorman (Eds.), Understanding language understanding: Computational models of reading (pp. 227-252). Cambridge, MA: MIT Press.

Meyer, B. J. F., \& Freedle, R. O. (1984). Effects of discourse type on recall. American Educational Research Journal, 21, 121-143.

Myers, J. L., \& O'Brien, E. J. (1998). Accessing the discourse representation during reading. Discourse Processes, 26, 131-157.

Myers, J. L., O’Brien, E. J., Albrecht, J. E., \& Mason, R. A. (1994). Maintaining global coherence during reading. Journal of Experimental Psychology: Learning, Memory, \& Cognition, 20, 876-886.

O'Brien, E. J. (1995). Automatic components of discourse comprehension. In R. F. Lorch, Jr., \& E. J. O’Brien (Eds.), Sources of coherence in reading (pp. 159-176). Hillsdale, $\mathrm{NJ}$ : Erlbaum.

Peeck, J., van den Bosch, A. B., \& Kreupeling, W. J. (1982). Effect of mobilizing prior knowledge on learning from text. Journal of Educational Psychology, 74, 771-777.

Posner, G., Strike, K., Hewson, P., \& Gertzog, W. (1982). Accom- modation of a scientific conception: Toward a theory of conceptual change. Science Education, 66, 211-227.

Pressley, M., \& Afflerbach, P. (1995). Verbal protocols of reading: The nature of constructively responsive reading. Hillsdale, NJ: Erlbaum.

PRITCHARD, R. (1990). The effects of cultural schemata on reading processing strategies. Reading Research Quarterly, 24, 273-295.

Rapp, D. N., Gerrig, R. J., \& Prentice, D. A. (2001). Readers' traitbased models of characters in narrative comprehension. Journal of Memory \& Language, 45, 737-750.

Rapp, D. N., \& VAN DEN Broek, P. (2005). Dynamic text comprehension. Current Directions in Psychological Science, 14, 276-279.

RECHT, D. R., \& LESLIE, L. (1988). Effect of prior knowledge on good and poor readers' memory of text. Journal of Educational Psychology, 80, 16-20.

Singer, M., Andrusiak, P., Reisdorf, P., \& Black, N. L. (1992). Individual differences in bridging inference processes. Memory \& Cognition, 20, 539-548.

Trabasso, T., \& SuH, S. (1993). Understanding text: Achieving explanatory coherence through on-line inferences and mental operations in working memory. Discourse Processes, 16, 3-34.

TuKEY, J. W. (1977). Exploratory data analysis. Reading, MA: AddisonWesley.

VAN Den Broek, P., Fletcher, C. R., \& Risden, K. (1993). Investigations of inferential processes in reading: A theoretical and methodological integration. Discourse Processes, 16, 169-180.

VAN DEN Broek, P., \& Kremer, K. E. (1999). The mind in action: What it means to comprehend during reading. In B. Taylor, M. Graves, \& P. van den Broek (Eds.), Reading for meaning (pp. 1-31). New York: Teacher's College Press.

van den Broek, P., Rapp, D. N., \& Kendeou, P. (2005). Integrating memory-based and constructionist processes in accounts of reading comprehension. Discourse Processes, 39, 299-316.

van den Broek, P., Risden, K., \& Husebye-Hartmann, E. (1995). The role of readers' standards of coherence in the generation of inferences during reading. In R. F. Lorch, Jr., \& E. J. O'Brien (Eds.), Sources of coherence in reading (pp. 353-373). Hillsdale, NJ: Erlbaum.

van den Broek, P., Young, M., Tzeng, Y., \& Linderholm, T. (1999). The Landscape model of reading: Inferences and the online construction of memory representation. In H. van Oosterndorp \& S. R. Goldman (Eds.), The construction of mental representations during reading (pp. 71-98). Mahwah, NJ: Erlbaum.

ZWAAN, R. A. (1999). Five dimensions of narrative comprehension: The events indexing model. In S. R. Goldman, A. C. Graesser, \& P. van den Broek (Eds.), Narrative comprehension, causality, and coherence (pp. 93-110). Mahwah, NJ: Erlbaum.

ZwAAN, R. A., \& BRown, C. M. (1996). The influence of language proficiency and comprehension skill on situation-model construction. Discourse Processes, 21, 289-327.

ZwaAn, R. A., \& Singer, M. (2003). Text comprehension. In A. C. Graesser, M. A. Gernsbacher, \& S. R. Goldman (Eds.), Handbook of discourse processes (pp. 83-121). Hillsdale, NJ: Erlbaum.

\section{NOTES}

1. We thank an anonymous reviewer for pointing this out. 2. We thank Joe Magliano for this suggestion.

(Manuscript received March 22, 2006; revision accepted for publication December 19, 2006.) 